\title{
Coherent random lasers in weakly scattering polymer films containing silver nanoparticles
}

\section{$\operatorname{AUTHOR}(S)$ :}

Meng, Xiangeng; Fujita, Koji; Murai, Shunsuke; Tanaka, Katsuhisa

\section{CITATION:}

Meng, Xiangeng ... [et al]. Coherent random lasers in weakly scattering polymer films containing silver nanoparticles. PHYSICAL REVIEW A 2009, 79(5): 053817.

\section{ISSUE DATE:}

2009-05

URL:

http://hdl.handle.net/2433/109867

RIGHT:

(C) 2009 The American Physical Society 


\title{
Coherent random lasers in weakly scattering polymer films containing silver nanoparticles
}

\author{
Xiangeng Meng, ${ }^{1}$ Koji Fujita, ${ }^{1,2, *}$ Shunsuke Murai, ${ }^{1}$ and Katsuhisa Tanaka ${ }^{1}$ \\ ${ }^{1}$ Department of Material Chemistry, Graduate School of Engineering, Kyoto University, Katsura, Nishikyo-ku, Kyoto 615-8510, Japan \\ ${ }^{2}$ PRESTO, Japan Science and Technology Agency (JST), 4-1-8, Honcho Kawaguchi, Saitama 332-0012, Japan
}

(Received 13 November 2007; published 8 May 2009)

\begin{abstract}
We report on the observations of coherent random lasers in weakly scattering polymer films embedded with silver nanoparticles. The dominant oscillation cavity calculated from the ensemble-averaged power Fourier transform spectrum of single-shot emission spectra is shorter by more than two orders of magnitude than the scattering mean-free path, indicating that the oscillation cavity is loosely associated with multiple scattering. We suggest that the coherent feedback arises from the cooperative effect of light scattering and field enhancement in the vicinity of silver nanoparticles.
\end{abstract}

DOI: 10.1103/PhysRevA.79.053817

PACS number(s): 42.55.Zz, 42.25.Dd

\section{INTRODUCTION}

Random lasers have drawn considerable attention in recent years because they theoretically concern a very important physical issue, i.e., Anderson localization of photons, and experimentally lead to mirrorless laser devices with potential applications in displays, sensors, and biomedicine $[1,2]$. Random lasers have brought about new challenges to conventional laser theories since some phenomena cannot be interpreted by the traditional theories [3]. Although early researches focused on incoherent random lasers [4-8], a coherent random laser was first demonstrated in strongly scattering semiconductor powders [9]. To date, coherent random lasers have been achieved in various amplifying random media operating in both weakly and strongly scattering regimes [9-12]. In the meantime, the origin of laser spikes is still an open question even now. For strongly scattering systems, the photon localization is the most popular model responsible for the emergence of coherent random lasers [9]. In contrast, various models have been proposed to explain coherent random lasers occurring in diffusive or weakly scattering regimes such as absorption-induced localization [13], amplified extended modes [10], and so on.

Experimentally, amplifying random media have been made by combining two kinds of materials which give rise to multiple scattering and optical gain, respectively. Optical gain is supplied with laser dyes, laser crystals, or semiconductors, while dielectric materials with high refractive index (e.g., $\mathrm{ZnO}$ [14], $\mathrm{TiO}_{2}$ [15], and $\mathrm{GaP}[16]$ ) are used as scattering centers in the past decades. Metal nanoparticles, however, have received little attention as scattering centers in random lasers, although they possess attractive properties beneficial to the realization of random lasers as mentioned below. First, metal nanoparticles usually have larger scattering cross section $\sigma_{s}$ than dielectric nanoparticles to visible light when they have the same dimensions. The difference in $\sigma_{s}$ is especially noticeable for particles less than $100 \mathrm{~nm}$; while dielectric nanoparticles only show weak Rayleigh scattering, metals strongly scatter visible light due to the localized surface-plasmon resonance (LSPR). Second, the scatter-

\footnotetext{
*fujita@dipole7.kuic.kyoto-u.ac.jp
}

ing properties of metal nanoparticles are very sensitive to particle size and morphology. This character is very useful for the manipulation of scattering properties by means of these two parameters. Third, the electric field can be confined in the vicinity of the surface of metal nanoparticles due to LSPR; as a consequence, such confinement can be very effective for the excitation of active centers to provide high optical gain for laser oscillation. Metal nanostructures have been demonstrated to significantly enhance the spontaneous emission rate of semiconductor quantum wells [17], rareearth ions [18], and laser dyes [19] because of the interaction between emission centers and surface plasmons. Utilizing these advantages, some researchers reported random lasers mediated by metal nanoparticles with incoherent emissions $[20,21]$. They observed narrowing of an emission spectrum to several nanometers in width accompanied with a nonlinear increase in emission intensity, which are typical characteristics of incoherent random lasers. Recently, we have demonstrated a random laser with ultranarrow emission spikes ( $<0.1 \mathrm{~nm})$ from a silver $(\mathrm{Ag})$-nanoparticles-dispersed amplifying random medium, indicating a coherent laser emission [22]. In that report, the average size of $\mathrm{Ag}$ nanoparticles is $\sim 2 \mathrm{~nm}$ in diameter, so that the scattering cross section is much smaller than the absorption cross section $\sigma_{a}$. We proposed that the strong excitation of dye molecules in the vicinity of Ag nanoparticles due to the confinement of the electric field played an important role in the occurrence of laser spikes.

In this work, we have fabricated an amplifying random medium containing Ag nanoparticles with a larger size $(\sim 50 \mathrm{~nm}$ in diameter) and demonstrated that coherent random laser emissions around $570 \mathrm{~nm}$ can be attained in rather dilute random media of highly transparent polymer films with embedded $\mathrm{Ag}$ nanoparticles. For such sized $\mathrm{Ag}$ nanoparticles, the value of $\sigma_{s}$ is in the same order as $\sigma_{a}$ so that a significant contribution is expected from light scattering. A single sharp emission peak with full width at half maximum (FWHM) less than $0.2 \mathrm{~nm}$ appears at a threshold pump energy, while further increase in the pump energy brings about multiple laser spikes. For comparison, we have prepared two other films embedded with $\mathrm{TiO}_{2}$ nanoparticles $(\sim 50 \mathrm{~nm}$ in diameter) to have the same number density of particles $\rho$ and scattering mean-free path $l_{s}$, respectively, as those of $\mathrm{Ag}$ dispersed film, and found that the Ag-dispersed one has a 
lasing threshold lower than those of the $\mathrm{TiO}_{2}$-dispersed ones. We have examined the resonant cavity by calculating the ensemble-averaged power Fourier transform (PFT) spectrum of single-shot emission spectra and found that the dominant cavity was shorter by more than 2 orders of magnitude than $l_{s}$, implying that the oscillation cavity is loosely associated with multiple scattering. Meanwhile, single-shot spectra upon repeated pump pulses have shown little chaotic behaviors, which means that the observed lasing spikes cannot be explained by amplified extended modes. We suggest that the coherent feedback may arise from the cooperative effect of light scattering and field enhancement in the vicinity of Ag nanoparticles. We expect that this work will inspire extensive studies in the field of metal nanoparticles-mediated random lasers.

\section{SAMPLES AND EXPERIMENTAL SETUP}

Our amplifying random medium was a $100-\mu$ m-thick poly(methyl methacrylate) (PMMA) film containing 3 $\times 10^{-3}$ vol \% Ag nanoparticles and $10 \mathrm{mM}$ rhodamine $6 \mathrm{G}$ (R6G). Ag nanoparticles were synthesized through a twostep seeded growth procedure reported previously [23]. A seed solution containing ultrafine Ag nanoparticles was prepared by adding silver nitrate and trisodium citrate into 20 $\mathrm{ml}$ of deionized water to make the final concentrations of $0.25 \mathrm{~m} M$ and $0.25 \mathrm{~m} M$, respectively. Then, $0.6 \mathrm{ml}$ of $10 \mathrm{~m} M$ sodium borohydride aqueous solution was added into the above solution all at once under vigorous stirring. After $2 \mathrm{~h}$, the seed solution was used to prepare Ag nanoparticles. A growth solution was prepared by mixing $10 \mathrm{ml}$ of $5 \mathrm{~m} M$ silver nitrate aqueous solution, $10 \mathrm{ml}$ of $1 \mathrm{~m} M$ tannin aqueous solution, and $10 \mathrm{ml}$ of as-prepared seed solution. The mixture was gently shaken for several minutes to make it homogeneous. After the resultant solution was aged for 12 h, Ag nanoparticles were collected by centrifugation. The distribution of size and morphology for Ag nanoparticles was checked by a field-emission scanning electron microscope [(FE-SEM) JSM-6700F, JEOL Ltd.]. Optical-absorption spectra were measured with a JASCO-V570 UV-visible-near infrared spectrophotometer.

The PMMA-Ag-R6G film was prepared by the cellcasting technique from a chloroform solution where PMMA and R6G were dissolved and Ag nanoparticles were ultrasonically dispersed. The $l_{s}$ of the film was estimated to be $\sim 3.4 \mathrm{~mm}$ for $\lambda=570 \mathrm{~nm}$ by the formula $l_{s}=1 / \rho \sigma_{s}$ [24], where $\rho=4.58 \times 10^{17} \mathrm{~m}^{-3}$ and $\sigma_{s}=6.38 \times 10^{-16} \mathrm{~m}^{2}$ was calculated for a $50 \mathrm{~nm}$ diameter Ag sphere in the PMMA host using the Mie theory. For comparison of random lasing properties, $100-\mu \mathrm{m}$-thick PMMA-R6G films containing $50 \mathrm{~nm}$ diameter $\mathrm{TiO}_{2}$ nanoparticles (rutile, TTO-55C, Ishihara, Japan) were prepared by the above-mentioned procedure. Two kinds of film were prepared; one with the same $\rho\left(l_{s}\right.$ $=59.2 \mathrm{~mm}$ ) as that of PMMA-Ag-R6G film and the other with the same $l_{s}\left(\rho=7.97 \times 10^{18} \mathrm{~m}^{-3}\right)$ as the PMMA-AgR6G film, respectively, while the concentration of R6G was the same as that in the PMMA-Ag-R6G film. $\mathrm{TiO}_{2}$ nanoparticles were chosen as scattering centers for comparison because of their high refractive index $(n \sim 2.7$ for rutile) lead- ing to a large contrast with PMMA $(n \sim 1.5)$, which is favorable for observation of coherent random lasers.

In experiments of random lasers, the film sample on a three-dimensional stage was optically pumped by the second harmonic of a mode-locked $\mathrm{Nd}^{3+}$ : yttrium aluminum garnet $\left(\mathrm{Nd}^{3+}\right.$ : YAG) laser $(532 \mathrm{~nm}, 10 \mathrm{~Hz}$ repetition rate, and $25 \mathrm{ps}$ pulse duration). The laser beam (6.0 mm diameter) was focused on the sample through a cylindrical lens to form a stripe geometry on the sample surface. The stripe width was $\sim 17 \mu \mathrm{m}$, while the stripe length could be varied from 0.1 to $6 \mathrm{~mm}$ by moving the stage. The emission was collected along the stripe with a fiber device into a monochromator (SPEX 270M) coupled to a charge couple device [(CCD) 3000 , Jobin Yvon, the spectral resolution is $0.07 \mathrm{~nm}$ ] cooled with liquid nitrogen.

\section{RESULTS AND DISCUSSION}

Figure 1(a) presents the FE-SEM image of Ag nanoparticles, clearly showing a somewhat wide distribution in both size and morphology. The average size of Ag nanoparticles is $\sim 50 \mathrm{~nm}$ in diameter. The shape of Ag particles is not spherical but polyhedral. The Ag nanoparticles exhibit intense LSPR signals over the whole visible wavelength region with two main peaks at 425 and $540 \mathrm{~nm}$ along with a shoulder at $360 \mathrm{~nm}$, as shown in Fig. 1(b) (curve 1). At the emission wavelength of R6G of around $570 \mathrm{~nm}$, the extinction is still quite intense, indicative of a considerable interaction between the emission of dye molecules and surface plasmons of the Ag nanoparticles. Also shown in Fig. 1(b) is a theoretical extinction curve calculated using the Mie theory for a 50-nm-diameter Ag sphere in water (curve 2). The main peak at $425 \mathrm{~nm}$ and shoulder at $360 \mathrm{~nm}$, corresponding to dipole and quadropole components of the resonance, respectively, are qualitatively reproduced. The peak at $540 \mathrm{~nm}$ in experimental curve is probably due to the resonance along the longer axis of anisotropic Ag particles. The experimental curve is much broader than the theoretical one, indicating a wide distribution in particle size and shape. Figure 1(c) shows the evolution of emission spectra against the pump energy. When the pump energy is low, the emission spectrum exhibits a broad band centered at $\sim 590 \mathrm{~nm}$ with FWHM of $\sim 46 \mathrm{~nm}$, which can be ascribed to the spontaneous emission of R6G. As the pump energy is increased up to $0.36 \mu \mathrm{J}$ (corresponding to an excitation energy intensity $0.14 \mathrm{MW} / \mathrm{mm}^{2}$ ), a single sharp peak at $572 \mathrm{~nm}$ with FWHM less than $0.2 \mathrm{~nm}$ suddenly emerges in the spontaneous emission band, indicating that the system becomes unstable and lasing occurs upon this pump energy. Above this pump energy, the emission intensity increases more rapidly, as shown in Fig. 1(d), indicating the occurrence of laser oscillation. In contrast, a sample without Ag nanoparticles merely shows a spontaneous emission band with FWHM of $\sim 46 \mathrm{~nm}$ under the same pumping conditions (not shown here). An increase in the pump energy gives rise to discrete laser spikes, as illustrated in Fig. 1(c). The lasing frequencies are found to vary with the irradiated position on the sample, which is relevant to the randomness in spatial distribution of Ag nanoparticles in PMMA host. For PMMA-TiO $2-R 6 G$ 

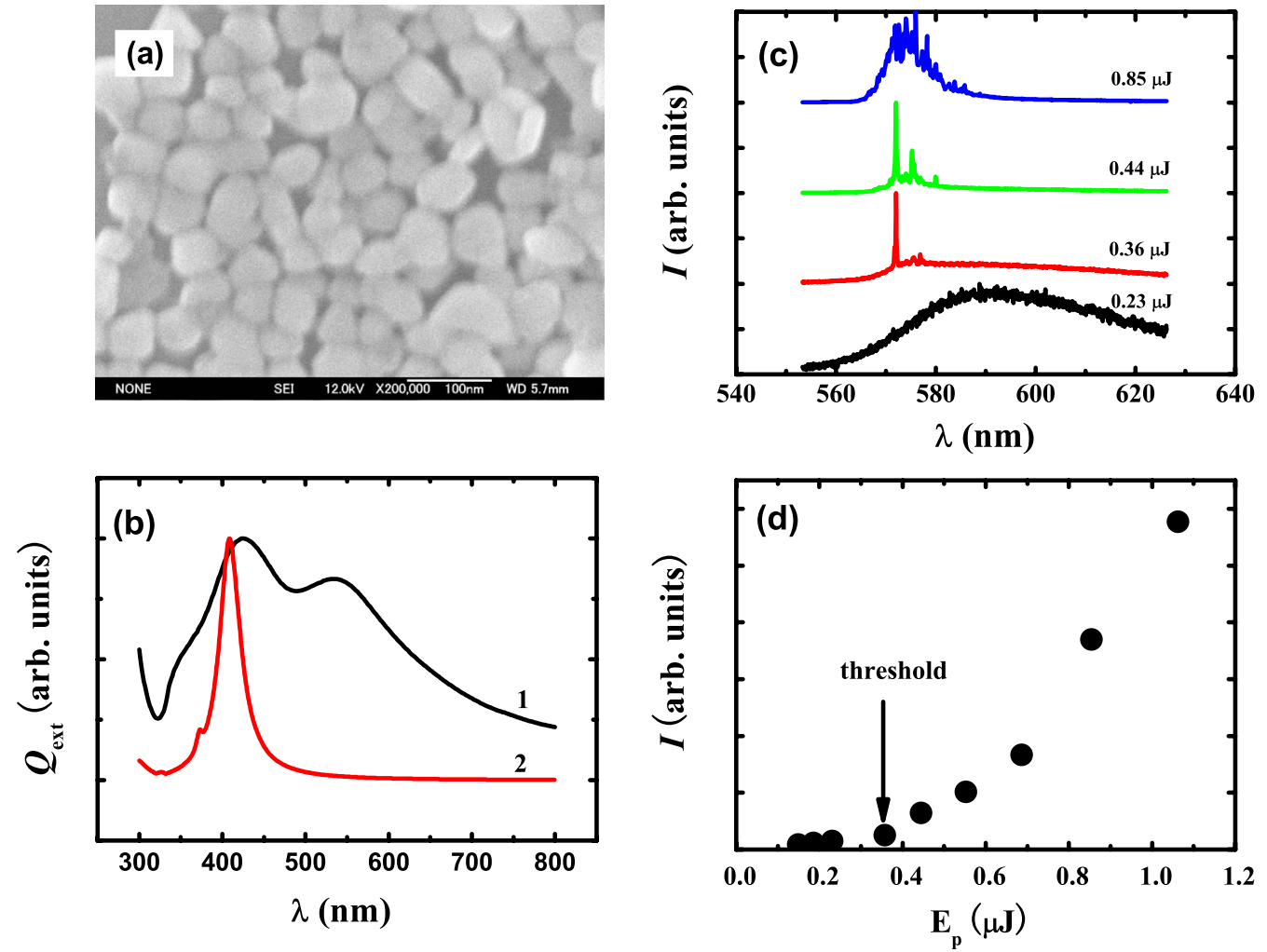

FIG. 1. (Color online) (a) FE-SEM image for Ag nanoparticles. (b) Experimental extinction spectrum of Ag nanoparticles dispersed in water (curve 1), showing two main bands at 425 and $540 \mathrm{~nm}$ along with a shoulder at $340 \mathrm{~nm}$. The theoretical extinction spectrum calculated by the Mie theory for a 50-nm-diameter Ag sphere suspended in water is shown for comparison (curve 2). Both of the spectra are normalized to unity. (c) Variation in the emission spectrum with the pump energy. The emission spectra were obtained under pump energies of $0.23,0.36$, 0.44 , and $0.85 \mu \mathrm{J}$. The sample is a 100- $\mu \mathrm{m}$-thick PMMA film containing $3 \times 10^{-3}$ vol \% Ag nanoparticles and $10 \mathrm{~m} M$ R6G. (d) Dependence of integrated emission intensity on pump energy, where the lasing threshold is indicated by the arrow.

films, discrete peaks are not observed in emission spectra until the pump energy reaches $\sim 0.64 \mu \mathrm{J}$ for the one with the same $l_{s}$ and $\sim 0.9 \mu \mathrm{J}$ for the one with the same $\rho$; and thus a higher threshold is required even for random media using rutile $\mathrm{TiO}_{2}$ as scattering centers. Furthermore, the emission intensity of PMMA-TiO $2-\mathrm{R} 6 \mathrm{G}$ films is much weaker than that of the PMMA-Ag-R6G film. The lower threshold and stronger emission intensity for the PMMA-Ag-R6G film probably arise from the contribution of LSPR. The comparison indicates that metal nanoparticles are preferable scattering elements toward the design of random lasers with high intensity and low threshold.

Although the system does not contain any external cavity, the lasing output is found to be highly unidirectional along the stripe direction. When the pump energy is just above the threshold, a single bright emission spot is observed by eyes on the screen normal to the stripe, while the increase in the pump energy causes the development into multiple sporadic spots. Figure 2(a) depicts the dependence of emission intensity on the probe angle, clearly showing that the emission is strongly confined in an angle range of $\pm 10^{\circ}$ from the direction of the stripe. Out of this angle range, what has been detected is not laser signals but spontaneous or amplified spontaneous emissions (ASEs). This fact indicates that the light amplification dominantly occurs along the long axis of the stripe. For instance, the emission collected along the stripe is a coherent laser emission, while the emission collected at $45^{\circ}$ away from the stripe is a broad band, as presented in Fig. 2(b). The absorption lengths at 532 and 570 $\mathrm{nm}$ are calculated to be $l_{a} \sim 6 \mu \mathrm{m}$ and $81 \mu \mathrm{m}$ through transmission measurements, respectively. The scattering meanfree path of $\sim 3.4 \mathrm{~mm}$ for $\lambda=570 \mathrm{~nm}$ means that both the penetration length of the pump beam and the stripe width are much shorter than $l_{s}$. In contrast, the stripe length can be tuned to several millimeters so that the optical gain will be much larger than the other dimensions and results in highly directional lasing output. It is noted that the waveguide mode reported for some thin-film random systems doped with dye could contribute to the unidirectional emission [25,26]. However the present sample is optically thick $(100 \mu \mathrm{m})$ and can sustain many modes, and so selective excitation of a specific mode is physically implausible. We have preliminarily checked this by measuring the polarization of emission and found no strong polarization for the emission.

For the purpose of exploring the effect of stripe length on lasing features, we have changed the irradiated stripe length while keeping the excitation energy density constant. Figure 2(c) depicts the evolution of emission spectra as a function of the stripe length under the excitation energy density of $1.3 \mathrm{MW} / \mathrm{mm}^{2}$. The number of lasing modes rapidly increases when the stripe length increases from 0.6 to $2.4 \mathrm{~mm}$. The increase in lasing modes with increasing excitation area 

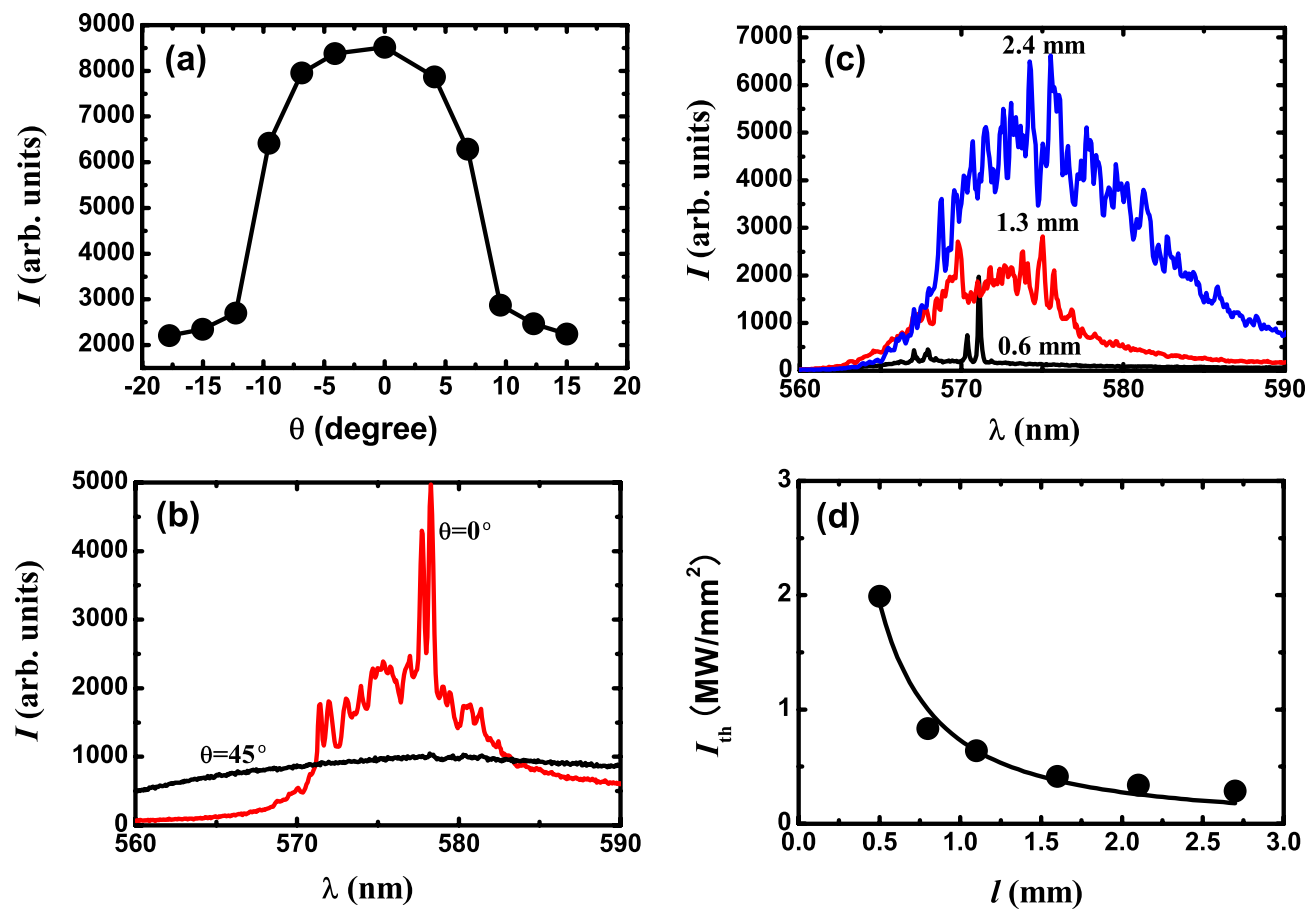

FIG. 2. (Color online) (a) Angular dependence of the emission intensity when the pump energy is $0.69 \mu \mathrm{J}$. (b) Emission spectra collected along the stripe $\left(\theta=0^{\circ}\right)$ and $45^{\circ}$ away from the stripe $\left(\theta=45^{\circ}\right)$. (c) Dependence of emission spectra on stripe length $l=0.6,1.3$, and $2.4 \mathrm{~mm}$. The excitation energy density is $1.3 \mathrm{MW} / \mathrm{mm}^{2}$. (d) Dependence of lasing threshold on the stripe length. The solid line represents a fit of $I_{\mathrm{th}}=a l^{b}$ to the experimental data, where $a=0.73 \pm 0.060$ and $b=-1.4 \pm 0.14$.

was also reported in literatures $[9,27]$. This phenomenon is explained by the increase in the number of oscillation cavities when the stripe length increases. The broad emission background centered at $\sim 585 \mathrm{~nm}$ is strongly inhibited at the stripe length of $0.6 \mathrm{~mm}$ but revives as the stripe length increases. In the present sample, since the emitted light far from the output end is more reabsorbed by dye molecules than that near the output end, the emission spectrum beyond the threshold pump energy is usually characterized by sharp spikes superimposed on a broad background. The reabsorption becomes more obvious as the stripe length increases, which can account for the revival of the broad background as observed in Fig. 2(c). The redshift in the emission spectrum with an increase in the stripe length is also caused by reabsorption of emitted light; as there is a significant overlap between the red side of the absorption spectrum and the blue side of the emission spectrum for R6G, a portion of emitted light in the blue side is reabsorbed by the R6G molecules. The increase in the stripe length increases the amount of reabsorbed light, resulting in the redshift in the emission spectrum. Figure 2(d) depicts the dependence of the threshold pump energy on the stripe length, showing that the threshold pump energy tends to decline with increasing the stripe length. Also shown in Fig. 2(d) is the curve drawn so as to fit to the experimental data. The curve abides by $I_{\text {th }}$ $=a l^{b}$ with $a=0.73 \pm 0.060$ and $b=-1.4 \pm 0.14$, where $I_{\text {th }}$ and $l$ are the threshold excitation energy density and stripe length, respectively. Considering that the excitation area $A$ $\propto l$, we obtain that $I_{\mathrm{th}} \sim A^{-1.4}$, in contrast to the theoretical relation of $A^{-0.75}$ [27]. It is probably because that our system cannot be described by the diffusion model.
The amplifying random media are either dynamic or static, depending on the time variance of configuration of scattering centers. A typical example of the former is the suspension of particles in a dye solution, where the particles can move ceaselessly. The ceaseless movement of the particles may result in the transient variation in the oscillation cavity for random lasers; therefore, it is reasonable to see pulse-to-pulse variation in lasing frequencies from singleshot emission spectra [11]. In contrast, for static random lasing systems (e.g., solid systems with embedded scattering centers), the frequencies of laser modes should be maintained from pulse to pulse since the static configuration of particles does not alter oscillation cavities for random lasers. However, the shot-to-shot variation or chaotic behavior in emission spectra was observed when coherent random lasers arose from noise amplification, in which a photon staying anomalously long time inside the active volume picks up a large gain [28]. Figure 3 represents six of single-shot emission spectra collected at the same pump position on the sample when the sample is irradiated with repeated pump pulses. Random laser emissions displayed in Fig. 3 do not show strongly chaotic behavior, in contrast to what has been reported in statically disordered media [28] but tend to populate in dominant locations that are denoted by $P_{n}(n: 1 \sim 6)$, implying that the frequencies of random lasers in the present sample are determined by certainly fixed oscillation cavities in the PMMA host instead of extended modes. We have confirmed that these peaks do not stem from the Raman scattering by exciting the sample with the third harmonic of $\mathrm{Nd}^{3+}$ :YAG laser $(\lambda=355 \mathrm{~nm})$. The distinct peaks still appear as seen by the excitation with $532 \mathrm{~nm}$ pulses, indicating 


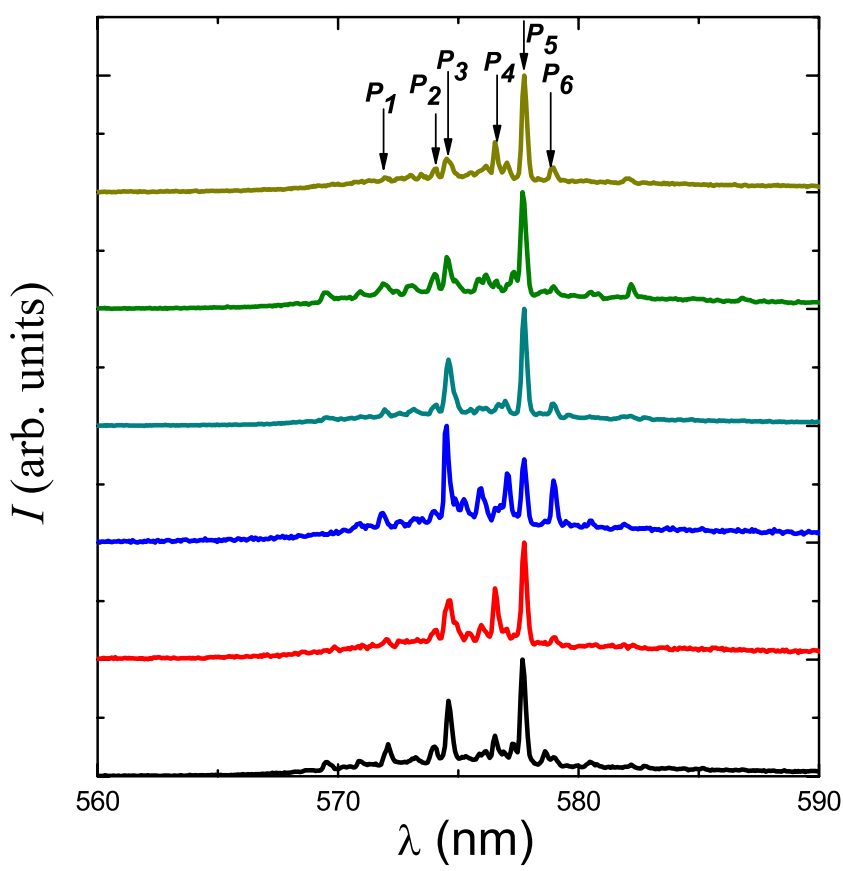

FIG. 3. (Color online) Six of single-shot emission spectra collected at the same position on the sample; the lasing modes tend to populate in frequencies denoted by $P_{n}(n: 1 \sim 6)$. The stripe length and width are $2.4 \mathrm{~mm}$ and $17 \mu \mathrm{m}$, respectively. The pump energy is about $0.85 \mu \mathrm{J}$.

that the observed peaks are not from Raman scattering but emission of dye molecules. The illuminated surface area $A$ is $4.1 \times 10^{4} \mu \mathrm{m}^{2}$ herein; so it can support lasing modes counting up to $N>7.8 \times 10^{5}$ estimated by $N=2 \pi A / \lambda^{2}$ [29] at $\lambda$ $=570 \mathrm{~nm}$. This large number combined with nonlinear effect leads to a strong-mode competition so that only the modes with longer lifetime can lase eventually.

In random lasers, if the laser oscillation results from multiple scattering of light, the lasing cavity length $L_{c}$ should be approximately integer times of $l_{s}$. As revealed previously $[11,30]$, the PFT spectrum of single-shot emission spectra offers the relationship between the Fourier transform components $d_{m}$ and $L_{c}$. A well-defined laser cavity exhibits peaks at $d_{m}$ that abides by $d_{m}=m L_{c} n / \pi$, where $m$ is an integer denoting the Fourier transform harmonics, and $n$ is the refractive index of the gain medium. To unveil the underlying resonators involved in random lasers, we calculated the ensembleaveraged PFT spectra from a sum of single-shot emission spectra along with the PFT spectra derived from individual single-shot emission spectra, as shown in Fig. 4. All the single-shot emission spectra involved in the calculation are collected at various pump positions on the sample since the Ag particle configuration is static and homogeneously dispersed in the film so that the cavity size is expected to be constant on average. The PFT spectra from individual singleshot emission spectra exhibit several distinctive peaks, as shown in Fig. 4(a), while the correlation between the individual spectra is not obvious. The underlying correlation becomes clear by the ensemble-averaged PFT spectra; rather than being averaged out and smoothed, the correlation in intensity increases and some peaks are observed with the

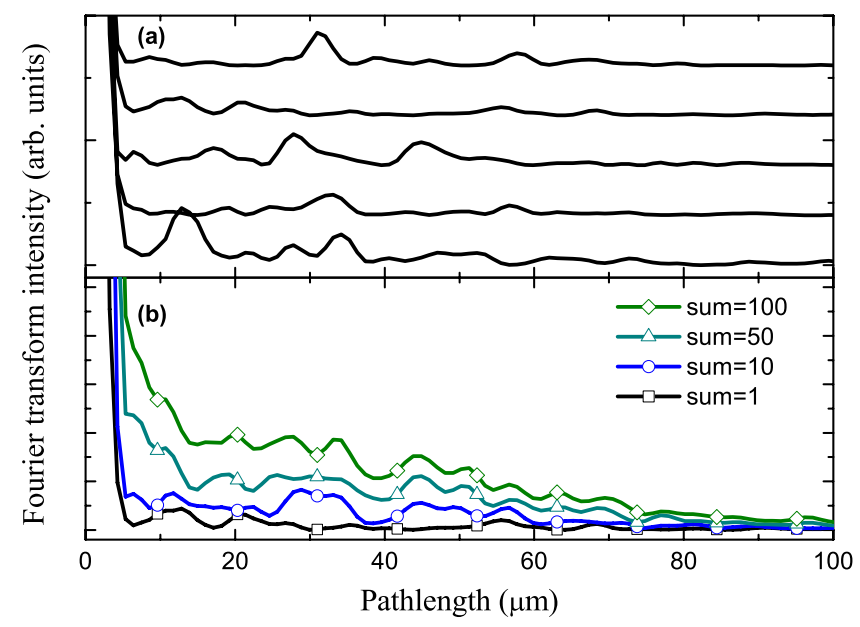

FIG. 4. (Color online) (a) Five of individual PFT spectra. (b) Ensemble-averaged PFT spectra of single-shot emission spectra collected at various sample positions upon the same pump energy. The number of single-shot emission spectra for ensemble-averaged PFT spectra is chosen as 10,50 , and 100 , respectively. An individual power Fourier transform spectrum is included for comparison. The stripe length under irradiation is fixed to be $2.4 \mathrm{~mm}$. The pump energy is set to be $0.85 \mu \mathrm{J}$.

increase in the number of single-shot emission spectra involved in the calculation. Hence, we utilize the ensembleaveraged PFT spectrum (calculated from 100 single-shot emission spectra here) to estimate the oscillation cavity length. The first peak at $\sim 6.4 \mu \mathrm{m}$ can be regarded as the fundamental Fourier component, while the others are regarded as the $m$ th harmonics. The oscillation cavity length is given by $L_{c}=\pi d_{1} / n$, so we obtain $L_{c}=13.0 \mu \mathrm{m}$ by using $n$ $=1.5$ for PMMA. The cavity length is shorter by more than two orders than $l_{s}(\sim 3.4 \mathrm{~mm})$, implying that the oscillation cavity is loosely associated with multiple scattering.

When we consider an amplifying random medium characterized by $l_{s}$, the sample may operate in three regimes, depending on the scattering strength. When $k l_{s} \leq 1$, where $k$ is the wave vector of the emitted photon and $k=2 \pi / \lambda$, the scattering strength is so strong that photon localization can be established via multiple scattering. Although the achievement of such a strong scattering has not been reported yet for visible light, some semiconductor-based random lasers with $k l_{s}$ value on the order of unity are argued to be operated in this scheme. As the scattering strength is decreased to a moderate scattering regime that satisfies $\lambda_{\mathrm{em}}<l_{s}<L$, where $\lambda_{\mathrm{em}}$ is the emission wavelength and $L$ is the sample size, the light propagation can be described by a normal diffusion process. Most of random lasers reported so far operate in this regime. As the scattering strength is decreased further until $l_{s}>L$, the sample falls in a sub-mean-free path regime (also called a ballistic regime). Since the sample in the present work is pumped by a stripe configuration and lasers are observed along the stripe length, the sample size can be characterized by the dimension along the stripe length and do not exceed the stripe length. Therefore, the scattering mean-free path is much larger than the sample size or the stripe length, indicating that the present sample operates in a sub-mean-freepath regime. 
Optical amplification in a sub-mean-free-path regime was first conducted by Kumar and co-workers [31] who proposed that the rare sub-mean-free path scattering events could significantly contribute to lasing despite of rather weakly scattering strength for an amplifying random medium. The probability of undergoing at least one scattering back in the finite amplifying random medium of size $L$ is given by $\left[1-\exp \left(-L / l_{t}\right)\right]$, where $l_{t}$ is a transport mean-free path after which the propagation direction of light is randomized. The corresponding gain factor is expressed by $\exp \left(L / l_{g}\right)$. Therefore, for the amplification to occur, the optical gain given by the product should exceed unity, i.e., $\left[1-\exp \left(-L / l_{t}\right)\right] \exp \left(L / l_{g}\right)>1$. Taking the absorption length into account, the effective $l_{t}$ is roughly estimated to be $\sim 81 \mu \mathrm{m}$ by means of $l_{t}^{-1}=l_{s}^{-1}+l_{a}^{-1}$. The sample size is set to be the possible minimum value of $81 \mu \mathrm{m}$ (the value of $l_{a}$ at $\lambda=570 \mathrm{~nm})$. The gain length is estimated by $l_{g}=1 / \rho \sigma_{\mathrm{em}}$, where $\rho$ and $\sigma_{\mathrm{em}}$ are the number density and emission cross section of R6G molecules, respectively. The value of $\rho$ is $\sim 6 \times 10^{24} \mathrm{~m}^{-3}$ since the concentration of R6G molecules is $10 \mathrm{~m} M$, while the value of $\sigma_{\mathrm{em}}$ is $\sim 2.0 \times 10^{-20} \mathrm{~m}^{2}$ [32]. This leads to $l_{g}=8 \mu \mathrm{m}$. Finally, the optical gain is calculated to be $\sim 620$ by taking $L=81 \mu \mathrm{m}, l_{t}=81 \mu \mathrm{m}$, and $l_{g}$ $=8 \mu \mathrm{m}$. The optical gain will increase further with the increase in the sample size $L$. The amplification is rather large so that coherent random lasers can be expected from the present weak scattering system. Therefore, even in such a weakly scattering system, the optical gain may trigger coherent lasing actions.

Another factor we should consider is that Ag nanoparticles are used as scattering centers instead of dielectric nanoparticles. Metal nanoparticles not only scatter but also absorb photons. Therefore, the role of optical absorption by Ag nanoparticles has to be taken into account when discussing the interaction between Ag nanoparticles and dye molecules. When the excitation pulse energy is absorbed by Ag nanoparticles to excite LSPR, the enhanced local electric field strongly excites dye molecules in the vicinity of par- ticles, which could provide dye molecules with gigantic gain. The extinction cross section $\sigma_{e}$ of one particle is a sum of $\sigma_{s}$ and $\sigma_{a}$; i.e., $\sigma_{e}=\sigma_{s}+\sigma_{a}$. The scattering cross section of a 50-nm-diameter $\mathrm{Ag}$ sphere is $\sigma_{s} \sim 6.38 \times 10^{-16} \mathrm{~m}^{2}$, while $\sigma_{a} \sim 1.44 \times 10^{-16} \mathrm{~m}^{2}$ at $\lambda=570 \mathrm{~nm}$, indicating that $\sigma_{s}$ is in the same order of $\sigma_{a}$. This is in sharp contrast to our previous report using very small particles (diameter $\sim 2 \mathrm{~nm}$ ), where $\sigma_{a}$ is 3 orders higher than $\sigma_{s}$. Therefore, the coherent feedback may arise from the cooperative effect of light scattering and field enhancement in the vicinity of Ag nanoparticles in the present work; but the effect of absorption is relatively small compared with that in our previous work.

\section{CONCLUSION}

In conclusion, we have observed coherent random lasers from rarely reported amplifying random media using metal nanoparticles as scattering centers. We found that the pump threshold was lowered and the emission intensity was enhanced by replacing $\mathrm{TiO}_{2}$ nanoparticles with the same sized Ag nanoparticles as scattering centers in PMMA-R6G films. Therefore, Ag nanoparticles are preferable scattering centers in the design of random lasers with high intensity and low threshold. The coherent feedback may arise from the cooperative contributions of light scattering and field enhancement in the vicinity of $\mathrm{Ag}$ nanoparticles. It would be interesting to make deep insight into the coherent mechanism in the future work. We expect that this work will result in extended studies in areas of random lasers induced by metal nanoparticles.

\section{ACKNOWLEDGMENTS}

This study was financially supported by a Grant in-Aid for Scientific Research (Grant No. 18360316) from the Ministry of Education, Culture, Sports, Science, and Technology, Japan.
[1] H. Cao, J. Phys. A 38, 10497 (2005).

[2] D. S. Wiersma, Nat. Phys. 4, 359 (2008).

[3] H. E. Türeci, L. Ge, S. Rotter, and A. D. Stone, Science 320, 643 (2008).

[4] N. M. Lawandy, R. M. Balachandran, A. S. L. Gomes, and E. Saultravioletain, Nature (London) 368, 436 (1994).

[5] C. Gouedard, D. Husson, C. Sauteret, F. Auzel, and A. Migus, J. Opt. Soc. Am. B 10, 2358 (1993).

[6] W. Sha, C. H. Liu, and R. Alfano, Opt. Lett. 19, 1922 (1994).

[7] M. A. Noginov, H. J. Caulfield, N. E. Noginova, and P. Venkateswarlu, Opt. Commun. 118, 430 (1995).

[8] M. Siddique, R. R. Alfano, G. A. Berger, M. Kempe, and A. Z. Genack, Opt. Lett. 21, 450 (1996).

[9] H. Cao, Y. G. Zhao, S. T. Ho, E. W. Seelig, Q. H. Wang, and R. P. H. Chang, Phys. Rev. Lett. 82, 2278 (1999).

[10] S. Mujumdar, M. Ricci, R. Torre, and D. S. Wiersma, Phys. Rev. Lett. 93, 053903 (2004).
[11] R. C. Polson and Z. V. Vardeny, Phys. Rev. B 71, 045205 (2005).

[12] X. Wu, W. Fang, A. Yamilov, A. A. Chabanov, A. A. Asatryan, L. C. Botten, and H. Cao, Phys. Rev. A 74, 053812 (2006).

[13] A. Yamilov, X. Wu, H. Cao, and A. L. Burin, Opt. Lett. 30, 2430 (2005).

[14] S. F. Yu and E. S. P. Leong, IEEE J. Quantum Electron. 40, 1186 (2004).

[15] G. van Soest, F. J. Poelwijk, R. Sprik, and A. Lagendijk, Phys. Rev. Lett. 86, 1522 (2001).

[16] K. L. van der Molen, R. W. Tjerkstra, A. P. Mosk, and A. Lagendijk, Phys. Rev. Lett. 98, 143901 (2007).

[17] K. Okamoto, I. Niki, A. Shvartser, Y. Narukawa, T. Mukai, and S. Scherer, Nature Mater. 3, 601 (2004).

[18] D. M. da Silva, L. R. P. Kassab, S. R. Luthi, C. B. De Araujo, A. S. L. Gomes, and M. J. V. Bell, Appl. Phys. Lett. 90, 081913 (2007). 
[19] K. Aslan, M. Wu, J. R. Lakowicz, and C. D. Geddes, J. Am. Chem. Soc. 129, 1524 (2007).

[20] G. Dice, S. Mujumdar, and A. Y. Elezzabi, Appl. Phys. Lett. 86, 131105 (2005).

[21] O. Popov, A. Zilbershtein, and D. Davidov, Appl. Phys. Lett. 89, 191116 (2006).

[22] X. Meng, K. Fujita, Y. Zong, S. Murai, and K. Tanaka, Appl. Phys. Lett. 92, 201112 (2008).

[23] X. Tian, W. Wang, and G. Cao, Mater. Lett. 61, 130 (2007).

[24] H. C. van de Hulst, Light Scattering by Small Particles (Dover, New York, 1957).

[25] S. V. Frolov, Z. V. Vardeny, K. Yoshino, A. Zakhidov, and R. H. Baughman, Phys. Rev. B 59, R5284 (1999).

[26] Q. Song, L. Wang, S. Xiao, X. Zhou, L. Liu, and L. Xu, Phys.
Rev. B 72, 035424 (2005).

[27] Y. Ling, H. Cao, A. L. Burin, M. A. Ratner, X. Liu, and R. P. H. Chang, Phys. Rev. A 64, 063808 (2001).

[28] S. Mujumdar, V. Türck, R. Torre, and D. S. Wiersma, Phys. Rev. A 76, 033807 (2007).

[29] J. H. Li and A. Z. Genack, Phys. Rev. E 49, 4530 (1994).

[30] R. C. Polson, A. Chipouline, and Z. V. Vardeny, Adv. Mater. (Weinheim, Ger.) 13, 760 (2001).

[31] B. R. Prasad, H. Ramachandran, A. K. Sood, C. K. Subramanian, and N. Kumar, Appl. Opt. 36, 7718 (1997).

[32] W. Holzer, H. Gratz, T. Schmitt, A. Penzkofer, A. Costela, I. García-Moreno, R. Sastre, and F. J. Duarte, Chem. Phys. 256, 125 (2000). 